\title{
Immune recovery and the risk of CMV/ EBV reactivation in children post allogeneic haematopoietic stem cell transplantation
}

\author{
MAEGORZATA JANECZKO, MONIKA MIELCAREK, BLANKA RYBKA, \\ RENATA RYCZAN-KRAWCZYK, DOROTA NOWOROLSKA-SAUREN, KRZYSZTOF KAEWAK
}

Department of Paediatric Haematology, Oncology, and Bone Marrow Transplantation, Wroclaw Medical University, Poland

\begin{abstract}
Immune reconstitution was studied prospectively in 86 children who underwent allogeneic haematopoietic stem cell transplantation (HSCT). We analysed the risk of cytomegalovirus (CMV) and Epstein-Barr virus $(E B V)$ reactivation in correlation with the kinetics of immune recovery and in relation to other potential risk factors that may influence the reactivation of these viruses including: diagnosis, type of HSCT, source of stem cells, type of conditioning, or the occurrence of graft-versus-host disease $(G v H D)$. The absolute number of lymphocyte subpopulations in peripheral blood was evaluated in seven timepoints following HSCT. Significantly lower values of both $\mathrm{CD}^{+}$and $\mathrm{CD}^{+} \mathrm{CD}^{+}$lymphocytes on day +14 and significantly higher values of both these subsets on day +168 post-transplant in patients with $C M V$ reactivation were observed. Significantly lower values of $C D 3^{+} C D 4^{+}$subpopulation were noted in patients with CMV reactivation on day +28 post allo-HSCT. Significantly lower lymphocyte values in the group with $E B V$ reactivation comparing with the group without $E B V$ reactivation were confirmed only in the case of pan-B lymphocytes $\left(C D 19^{+}\right)$subpopulation on day $+21,+28$, and +84 post allo-HSCT. We identified the impact of CMV reactivation on occurrence of the intestinal acute GvHD, which occurred more frequently in the group with CMV reactivation compared with patients without reactivation. Higher incidence of chronic GvHD was also observed in patients with CMV reactivation compared to the group without reactivation. EBV reactivation occurred more frequently in patients receiving transplants from matched unrelated donors, in particular after peripheral blood stem cell transplantation and while implementing antithymocyte globulin as GvHD prophylaxis.
\end{abstract}

Key words: immune recovery, CMV reactivation, EBV reactivation, allogeneic $H S C T, G v H D$, children.

(Cent Eur J Immunol 2016; 41 (3): 287-296)

\section{Introduction}

Haematopoietic stem cell transplantation (HSCT) remains a standard treatment for numerous malignant disorders as well as many non-malignant conditions, including metabolic disorders, primary immunodeficiencies, and bone marrow failure syndromes. The functions of the immune system are profoundly impaired for many months following HSCT, which is associated with an increased risk of opportunistic infections, viral reactivation, and graft-versus-host disease (GvHD). Major causes of morbidity and mortality in patients after allogeneic HSCT are: relapse of the underlying disease $(30 \%$ in patients transplanted from a matched family donor), GvHD (25\%), and bacterial, viral, or fungal infections following HSCT (11\%) [1]. The duration of a complete immune recovery of the haematopoietic system after allogenic HSCT
(allo-HSCT) may vary among patients, and its pace and quality is affected by various external and internal factors. The factors having the greatest influence on early recovery described in the literature are: the number of transplanted stem cells $\left(\mathrm{CD} 34^{+}\right)$and $\mathrm{T}$ lymphocytes $\left(\mathrm{CD}^{+}\right)$, the patient's age, cytomegalovirus (CMV) status of the donor and the recipient, type of transplant, conditioning regimen, T-cell depletion of bone marrow transplants, and the type of GvHD prophylaxis [2-4]. Immune reconstitution usually starts with the appearance of granulocytes, monocytes, macrophages, and NK lymphocytes in the bloodstream. Afterwards the subpopulations of $\mathrm{T}$ lymphocytes start to recover and finally B lymphocytes appear. Infections during the early post-transplant period (the aplastic phase) result from a deficiency in both the number and function of both granulocytes and mononu-

Correspondence: Krzysztof Kałwak, MD, PhD, Prof., Department of Paediatric Haematology/Oncology and BMT,

Wroclaw Medical University, Supraregional Centre of Paediatric Oncology "Cape of Hope", 213 Borowska St., $50-556$ Wroclaw, Poland, e-mail: ml.janeczko@gmail.com, krzysztof.kalwak@gmail.com

Submitted: 18.05.2016; Accepted: 27.07.2016

Central European Journal of Immunology 2016; 41(3) 
clear cells, and these are mostly bacterial or fungal infections [5]. Later on during the period from initial marrow engraftment to at least the third or fourth month infections are mainly because of cell-mediated immune deficiency with a decreased number and function of specific and non-specific cytotoxic cells [6]. During this phase the greatest problem is viral infections: both reactivations and new infections (i.e. CMV, Epstein-Barr virus (EBV), adenovirus (ADV), enteric and respiratory viruses). The occurrence and severity of acute GvHD (aGvHD) is the main factor delaying immune recovery and favouring infections in this phase. Careful monitoring (by PCR and/or pp65 antigenaemia in CMV infections) is crucial during this phase and enables early diagnosis and implementation of preemptive treatment. During the third phase, beginning at the fourth month, the immune recovery is mainly influenced by the presence and severity of chronic GvHD (cGvHD) and is mainly linked to immunoglobulin deficiency. Patients are at high risk of bacterial infections, in particular with encapsulated bacteria. The prevalence of post-transplant infections depends on the speed of immune recovery and appears to be linked to the intensity of immunosuppression and the implemented drugs, most probably by their impact on $\mathrm{T}$ cell number and function [7]. However, early viral infections may also have a direct effect on immune recovery after HSCT. Their incidence and treatment appears to be linked to the degree of immunosuppression and the speed of immune recovery following transplant [8]. Delayed immune reconstitution and the resulting opportunistic infections in children following HSCT are strongly associated with increased treatment-related morbidity and mortality (TRM). Major advances in the monitoring and treatment of viral infections after HSCT have been achieved in recent years. Among the viruses, CMV remains a major infectious complication, particularly in recipients who develop GvHD and are highly immunosuppressed (i.e. recipients of unrelated transplants, T-cell-depleted marrow, and high-dose steroids) [9]. Cytomegalovirus reactivation has been reported in 40-70\% of HSCT recipients who are seropositive or have a seropositive donor [7]. CMV viraemia can be associated with multi-organ disease including pneumonitis, hepatitis, colitis, and retinitis. Significant progress has been made in the diagnostics and prevention of CMV disease over the past decade. Allogenic HSCT recipients must be monitored with a sensitive method for CMV in peripheral blood at least weekly until day +100 after transplant or longer in the case of GvHD presence or CMV reactivation. The most commonly used method is detection of the CMV pp65 antigenaemia in leucocytes by immune staining with monoclonal antibodies [10]. Treatment mostly consists of preemptive strategy using intravenous ganciclovir or its oral pro-drug - valganciclovir. However, the pre-transplantation CMV serological status of the donor and the recipient remains an important risk factor for post-transplantation outcome de- spite the use of antiviral prophylaxis and preemptive therapy [9]. Epstein-Barr virus in immunocompetent humans causes a mild self-limiting disease during primary infection and persists for life in latently infected B cells. In immunosuppressed individuals EBV-infected B lymphocytes may proliferate rapidly, resulting in polyclonal or monoclonal post-transplant lymphoproliferative disease (EBV-PTLD). It is a life-threatening complication that may occur after HSCT with an incidence of $0.45-29 \%$ and is mainly observed after unrelated allogenic HSCT $[11,12]$. Symptoms usually develop between one and five months after transplant, in most cases during the third month post-transplant. They mainly consist of mononucleosis-like syndrome (early disease) or a lymphoma-like picture with lymphadenopathy and mass lesions (PTLD). EBV reactivation following HSCT is a major concern, especially during periods of lymphopaenia, and historically (before the introduction of Rituximab therapy) 11-26\% of transplant recipients developed EBV-related lymphoproliferative disease [13, 14]. Although PTLD was reported to occur in $1 \%$ of all transplantation cases, higher rates might be seen in the presence of specific risk profiles such as intensive immunosuppression or T-cell depletion [12]. The treatment strategy includes the reduction of immunosuppression, rituximab (anti CD-20 antibody) that is highly effective for EBV-associated PTLD, and/or infusion of EBV-specific cytotoxic T-cells [15, 16]. However, little is known about the immunological consequences of the therapy with both rituximab and EBV-specific cytotoxic T-cells in paediatric allogeneic HSCT [17, 18]. Viral infections, including most commonly CMV and EBV reactivation, affect both the clinical and immunological recovery following allogeneic HSCT. Early viral infections linked with prolonged T-cell immunodeficiency and thymic dysfunction may also indicate the presence of subclinical GvHD [8].

The aims of the study were:

1. Comparison of the profile of immune recovery in patients with CMV and/or EBV reactivation with the profile of immune recovery in patients in whom no reactivation was found.

2. Analysis of the impact of CMV and EBV reactivation on the profile of immune reconstitution.

3. Analysis of selected factors: type of disease, type of transplant (HLA ID SIB, MUD, HLA MM REL), type of transplant (MSDPBSCT, MUDPBSCT, MSDBMT, MUDBMT, HAPLOPBSCT), source of stem cells, conditioning regimen, GvHD prophylaxis, CMV and EBV serological status of both donor and recipient and the recipients age and sex, which may contribute to the risk of CMV/EBV reactivation with particular reference to the profile of immune recovery.

\section{Material and methods}

Immunological reconstitution was analysed prospectively in 86 children ( 55 boys, 31 girls) who underwent 
allogeneic haematopoietic stem cell transplantation (alloHSCT) in the Department of Paediatric Haematology, Oncology, and Bone Marrow Transplantation, Medical University of Wroclaw between March 2013 and December 2014. The detailed characteristics of the study group are presented in Table 1.

Fifty-nine patients underwent transplant from matched unrelated donors (MUD; 69\%), 18 from matched family donors (HLA ID SIB, 21\%), and nine from mismatched family donors (HLA MM REL, 10\%). The source of stem cells was peripheral blood $(83 \%)$ or bone marrow $(17 \%)$. The reason for HSCT was malignant disease in 64 children $(74 \%)$ or non-malignant disease in 22 children $(26 \%)$. Among malignant diseases patients with acute lymphoblastic leukaemia (ALL) accounted for 38\% of all transplants, acute myeloblastic leukaemia (AML): 20\%, myelodysplastic syndromes (MDS): 3\%, solid tumours (including neuroblastoma (NB), Wilms tumour (WT) and primitive neuroectodermal tumour (PNET)): 7\%, Hodgkin disease (HD): 2\%, non-Hodgkin's lymphomas (NHL): $1 \%$, and chronic myeloid leukaemia (CML): $1 \%$. Among non-malignant diseases immunodeficiencies and bone marrow failures including: paroxysmal nocturnal haemoglobinuria (PNH), chronic granulomatous disease (CGD), severe combined immunodeficiency (SCID), Blackfan-Diamond anaemia (BDA), lymphoproliferative syndrome (XLP), Wiskott-Aldrich disease, Fanconi anaemia (FA), and congenital megakaryocytic thrombocytopaenia (CAMT) accounted for $21 \%$ of all diseases, while severe aplastic anaemia (SAA) and metabolic diseases accounted for $5 \%$ and $1 \%$, respectively. We divided patients according to the type of conditioning following the criteria described by Bacigalupo et al. into four groups: myeloablative (MA), reduced intensity conditioning (RIC), non-myeloablative (NMA), and patients without conditioning [19]. Patients with MA conditioning amounted to $58 \%$, RIC $28 \%$, NMA $10 \%$, and without conditioning $4 \%$ of all children. In GvHD prophylaxis antithymocyte globulin (ATG) was administered in 58\% of patients, CAMPATH in $15 \%$, and both ATG and CAMPATH in $1 \%$. In $26 \%$ of children no prophylaxis was implemented. The median age of the patients at the time of HSCT was 8.6 years (range 4 months - 19.9 years). Analyses of both immune recovery and viral reactivation were carried out from the day of the transplantation until day +168 post alloHSCT. During the observation period 14 patients died $(16.2 \%)$. Immune recovery was monitored by determining the absolute number of cells per microlitre of peripheral blood (cells $/ \mu \mathrm{l})$ in established points in a time starting from day +14 until day +168 post HSCT. We monitored recovery of Pan-T lymphocytes $\left(\mathrm{CD}^{+}\right)$and their subpopulations: helper $\mathrm{T}$ lymphocytes $\left(\mathrm{CD}^{+} \mathrm{CD}^{+}\right)$, cytotoxic/ suppressor $\mathrm{T}$ lymphocytes $\left(\mathrm{CD} 3^{+} \mathrm{CD} 8^{+}\right)$, Pan-B lymphocytes $\left(\mathrm{CD} 19^{+}\right)$, and Pan-NK lymphocytes $\left(\mathrm{CD} 3^{-} \mathrm{CD}^{+} 6^{+}\right)$. The analysis of immune recovery after allo-HSCT was
Table 1. Characteristics of the study group

\begin{tabular}{|c|c|c|}
\hline \multirow{2}{*}{$\begin{array}{l}\text { Patients } \\
\text { Sex }\end{array}$} & \multicolumn{2}{|c|}{$N=86$} \\
\hline & $n$ & $\%$ \\
\hline Male & 55 & 64 \\
\hline Female & 31 & 36 \\
\hline Type of HSCT & $n$ & $\%$ \\
\hline MUD & 59 & 69 \\
\hline HLA ID SIB & 18 & 21 \\
\hline HLA MM REL & 9 & 10 \\
\hline Source of stem cells & $n$ & $\%$ \\
\hline Peripheral blood & 71 & 83 \\
\hline - after $\mathrm{T}$ cell depletion in vitro & 8 & 9 \\
\hline Bone marrow & 15 & 17 \\
\hline Diagnosis & $n$ & $\%$ \\
\hline Malignant (M) & 64 & 74 \\
\hline Acute lymphoblastic leukaemia (ALL) & 33 & 38 \\
\hline Acute myeloblastic leukaemia (AML) & 17 & 20 \\
\hline Solid tumours (NBL, WT, PNET) & 6 & 7 \\
\hline Chronic myeloid leukaemia (CML) & 1 & 1 \\
\hline Hodgkin lymphoma (HL) & 2 & 2 \\
\hline Non-Hodgkin lymphoma (NHL) & 1 & 1 \\
\hline Myelodysplastic syndrome (MDS) & 3 & 3 \\
\hline Xanthogranuloma juvenile & 1 & 1 \\
\hline Non malignant (NM) & 22 & 26 \\
\hline Severe aplastic anaemia (SAA) & 4 & 5 \\
\hline Metabolic diseases (WM) & 1 & 1 \\
\hline Immune deficiencies (ID*) & 17 & 20 \\
\hline \multicolumn{3}{|l|}{ Conditionning } \\
\hline Myeloablative (MA) & 50 & 58 \\
\hline Reduced-intensity conditioning (RIC) & 24 & 28 \\
\hline Non-myeloablative (NMA) & 8 & 10 \\
\hline None & 4 & 4 \\
\hline
\end{tabular}

Graft versus host disease (GvHD) prophylaxis

\begin{tabular}{lcc}
\hline ATG & 50 & 58 \\
\hline CAMPATH & 13 & 15 \\
\hline None & 22 & 26 \\
\hline ATG and CAMPATH & 1 & 1 \\
\hline
\end{tabular}

HLA ID SIB - HLA identical sibling; MUD - matched unrelated donor; HLA MM REL - HLA mismatched related donor; NBL - neuroblastoma; WT - Wilms tumour; PNET - primitive neuroectodermal tumour

performed at seven time points: $+14,+21,+28,+42,+56$, +84 , and +168 days post transplantation. To monitor CMV reactivation the CMV-EA test was used detecting CMV pp65 antigen. CMV reactivation was defined as the pres- 
ence of the antigen in peripheral blood (positive nucleus/ nuclei in MNC). Reactivation of EBV was monitored by performing quantitative determination of EBV DNA presence in the peripheral blood using PCR method. Patients were prospectively monitored once a week for CMV-EA and EBV DNA presence in the peripheral blood. The statistical analysis was prepared using STATISTICA 10.0. In order to evaluate the influence of CMV and EBV reactivation on immune recovery ANOVA test with repeated measures was used. Patients were divided into two groups: with CMV/EBV reactivation - 1 ; and without CMV/EBV reactivation $-0 . R 1$ parameter determined the increase of lymphocytes within a given time $($ day $+14,+21,+28,+42$, $+56,+84$, and +168 after allo-HSCT). Each lymphocyte subpopulation was analysed separately. In order to assess the significance of differences in mean values of $\mathrm{CD}^{+}$ lymphocytes in defined points in time between the group with CMV reactivation: 1 and without CMV reactivation: 0 and due to the lack of normal distribution in both populations a nonparametric $U$ Mann-Whitney test was used.

To analyse the impact of: disease (malignant, non-malignant), donor (HLA ID SIB, MUD, HLA MM REL), type of transplant (MSDPBSCT, MUDPBSCT, MSDBMT, MUDBMT, HAPLOPBSCT), source of stem cells (PB, BM), conditioning regimen (MA, RIC, NMA, without conditioning), GvHD prophylaxis (ATG, CAMPATH, both ATG and CAMPATH, without prophylaxis), CMV and EBV serological status of donor and recipient and recipients, sex, and age on the risk of CMV reactivation a qualitative $\chi^{2}$ Pearson test was used.

\section{Results}

Cytomegalovirus reactivation was confirmed in 31 patients during the study period of 168 days; EBV reactivation occurred in 34 patients while in two of them EBV-associated post-transplant lymphoproliferative disease (PTLD) was observed. Acute GvHD was observed in 50 patients. Most commonly it was a cutaneous aGvHD $(n=46)$. Gastrointestinal aGvHD was confirmed in 15 children, while liver was affected in six patients. In some children there was more than one organ affected. Among all patients with aGvHD 13 had CMV reactivation, $14 \mathrm{EBV}$ reactivation, 6 both CMV and EBV reactivation, while in 17 we observed neither CMV nor EBV reactivation. Detailed data are presented in Table 2.

Chronic GvHD occurred in eight patients and affected: skin, liver, lungs, intestines, or joints. In four children with cGvHD we confirmed CMV reactivation, in one EBV reactivation, in two both CMV and EBV reactivation, and in one patient we observed neither CMV nor EBV reactivation.

\section{Pan-T lymphocytes $\left(\mathrm{CD3}^{+}\right)$}

The significance of the interaction effect between an increase of lymphocyte count in time (R1) and CMV reacti- vation was confirmed using ANOVA test with repeated measures $(p=0.012)$ with a high observed power of the test accounting for 0.877 . The effect of CMV reactivation as a single factor was not significant $(p=0.911)$. Significantly lower values of $\mathrm{CD}^{+}$lymphocytes in patients with $\mathrm{CMV}$ reactivation on the day $+14(p=0.026)$ and significantly higher values of $\mathrm{CD}^{+}$lymphocytes in this group on day $+168(p=0.046)$ were confirmed using $U$ Mann-Whitney test (Fig. 1).

\section{Helper T lymphocytes $\left(\mathrm{CD3}^{+} \mathrm{CD4}^{+}\right)$}

In order to assess the significance of differences in mean values of this lymphocyte subset between the group with CMV reactivation: 1 and without CMV reactivation: 0 the $U$ Mann-Whitney test was implemented. Significantly lower values of $\mathrm{CD}^{+} \mathrm{CD}^{+}$subpopulation were observed in patients with CMV reactivation on day +28 post allo-HSCT ( $p=0.040$, Fig. 2).

\section{Cytotoxic/suppressor T lymphocytes $\left(\mathrm{CD3}^{+} \mathrm{CDB}^{+}\right)$}

Analysis of the variance with repeated measures in the case of cytotoxic/suppressor T lymphocytes showed similar results to the pan-T lymphocytes analysis. Significant interaction between an increase in lymphocyte count and CMV reactivation $(p=0.0004)$ with the observed power of 0.979 was proven. By using the $U$ Mann-Whitney test significantly lower values of $\mathrm{CD} 3^{+} \mathrm{CD} 8^{+}$lymphocyte subset on day +14 post allo-HSCT in patients with CMV reactivation $(p=0.033)$ and significantly higher values of this subpopulation in the group with CMV reactivation on day +168 post allo-HSCT ( $p=0.006)$ were observed (Fig. 3 ).

\section{Pan-B lymphocytes (CD19')}

In order to assess the significance of differences in mean values of pan-B lymphocytes in the defined points in time between the group with CMV reactivation: 1 and without CMV reactivation: 0 the $U$ Mann-Whitney test was used. Significantly higher values of CD19+ lymphocyte subpopulation in the group with CMV reactivation on day +14 post allogenic HSCT $(p=0.008)$ and significantly lower values of pan-B lymphocytes on day +28 post allogenic HSCT $(p=0.038)$ were observed in this group (Fig. 4).

\section{Pan-NK lymphocytes $\left(\mathrm{CD3}^{-} \mathrm{CD56}^{+}\right)$}

$U$ Mann-Whitney analysis of pan-NK lymphocytes did not show any significant differences between the values of lymphocyte subpopulations in the two groups (Fig. 5).

In order to assess the significance of differences in mean values of $\mathrm{CD}^{+}, \mathrm{CD}^{+} \mathrm{CD}^{+}, \mathrm{CD}^{+} \mathrm{CD}^{+}, \mathrm{CD} 19^{+}$, and $\mathrm{CD}^{-} \mathrm{CD}^{-} 6^{+}$lymphocyte subsets on day $+14,+21,+28,+42$, $+56,+84$, and +168 post-allogenic HSCT between the group with EBV reactivation: 1 and without EBV reactivation the $U$ Mann-Whitney test was used. Significantly higher lym- 
Table 2. Median values of lymphocytes subpopulations in defined time points in four groups of patients (CMV reactivation, EBV reactivation, CMV and EBV reactivation, without CMV/EBV reactivation)

\begin{tabular}{|c|c|c|c|c|c|c|c|c|}
\hline Lymphocytes subsets & CMV/EBV reactivation & +14 & +21 & +28 & +42 & +56 & +84 & +168 \\
\hline \multirow[t]{4}{*}{$\mathrm{CD}^{+}$} & CMV react. & 3.00 & 29.00 & 48.00 & 162.50 & 374.00 & 666.00 & 532.00 \\
\hline & EBV react. & 27 & 55 & 98 & 219 & 362 & 344 & 284.5 \\
\hline & $\mathrm{CMV}$ and EBV react. & 2 & 251 & 209 & 374 & 448 & 579 & 1141 \\
\hline & no react. & 50.5 & 118.5 & 273.5 & 302 & 308.5 & 393 & 555.5 \\
\hline \multirow[t]{4}{*}{$\mathrm{CD}^{+}{ }^{+} \mathrm{CD} 4^{+}$} & CMV react. & 0.00 & 20.50 & 37.00 & 52.50 & 100.00 & 79.00 & 160.00 \\
\hline & EBV react. & 1 & 3 & 11 & 84 & 86 & 65 & 105.5 \\
\hline & CMV and EBV react. & 1 & 64 & 34 & 50 & 49 & 68.5 & 204.5 \\
\hline & no react. & 5 & 24 & 95 & 87.5 & 58 & 85 & 156 \\
\hline \multirow[t]{4}{*}{$\mathrm{CD}^{+}{ }^{+} \mathrm{CD}^{+}$} & CMV react. & 0.00 & 9.50 & 19.00 & 80.50 & 223.00 & 558.00 & 446.00 \\
\hline & EBV react. & 8 & 27 & 71 & 73 & 130 & 247 & 159 \\
\hline & $\mathrm{CMV}$ and EBV react. & 1 & 157 & 167 & 345 & 446 & 498.5 & 920 \\
\hline & no react. & 14.5 & 57.5 & 141.5 & 122.5 & 113.5 & 129 & 263 \\
\hline \multirow[t]{4}{*}{$\mathrm{CD}_{19}{ }^{+}$} & $\mathrm{CMV}$ react. & 0.00 & 1.00 & 1.00 & 2.50 & 6.00 & 32.00 & 162.00 \\
\hline & EBV react. & 2 & 7 & 6 & 9 & 6 & 0.5 & 24.5 \\
\hline & CMV and EBV react. & 0 & 15 & 6 & 4 & 4 & 4 & 162.5 \\
\hline & no react. & 1 & 3.5 & 7.5 & 7.5 & 13.5 & 34 & 184.5 \\
\hline \multirow[t]{4}{*}{$\mathrm{CD}^{-}{ }^{-\mathrm{CD}} 56^{+}$} & CMV react. & 20.00 & 84.50 & 133.00 & 129.00 & 183.00 & 187.00 & 203.00 \\
\hline & EBV react. & 45 & 118 & 198 & 145 & 181 & 121 & 138 \\
\hline & CMV and EBV react. & 14 & 134 & 124 & 138 & 241 & 177 & 170.5 \\
\hline & no react. & 62.5 & 106 & 187.5 & 150.5 & 166 & 166 & 166 \\
\hline
\end{tabular}

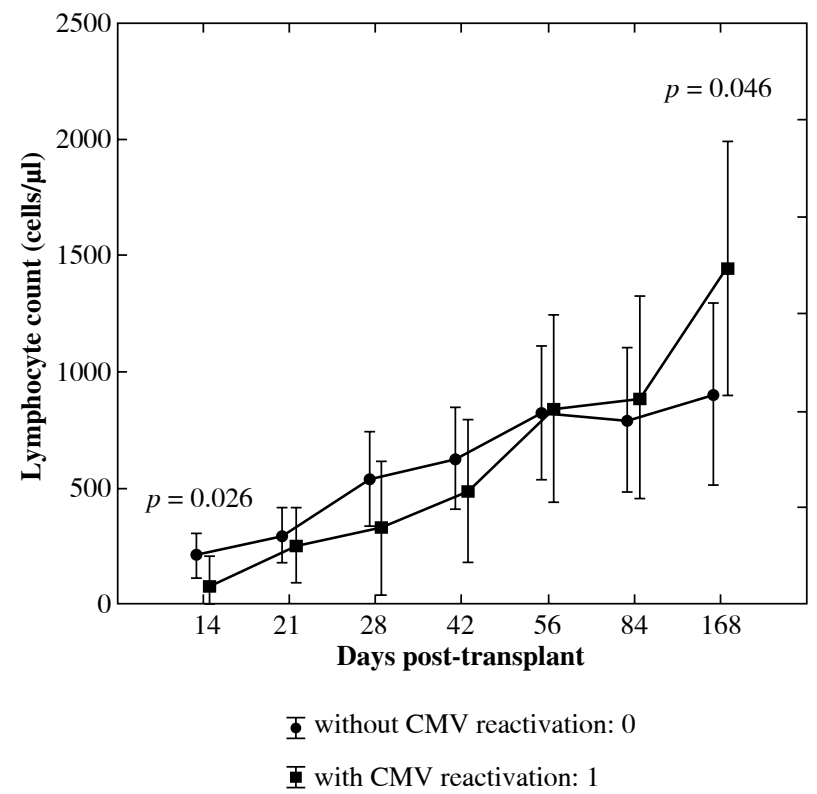

Fig. 1. Reconstitution of lymphocyte subsets after allogenic HSCT according to the occurrence of CMV reactivation - pan-T lymphocytes $\left(\mathrm{CD}^{+}\right)$

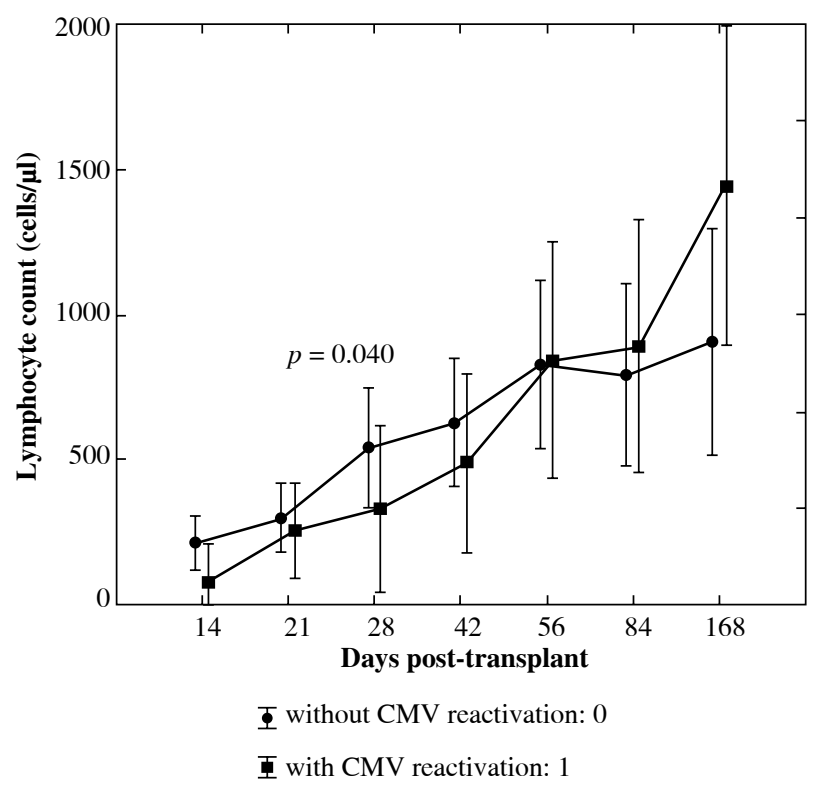

Fig. 2. Reconstitution of lymphocyte subsets after allogenic HSCT according to the occurrence of CMV reactivation - helper T lymphocytes $\left(\mathrm{CD} 3^{+} \mathrm{CD} 4^{+}\right)$ 


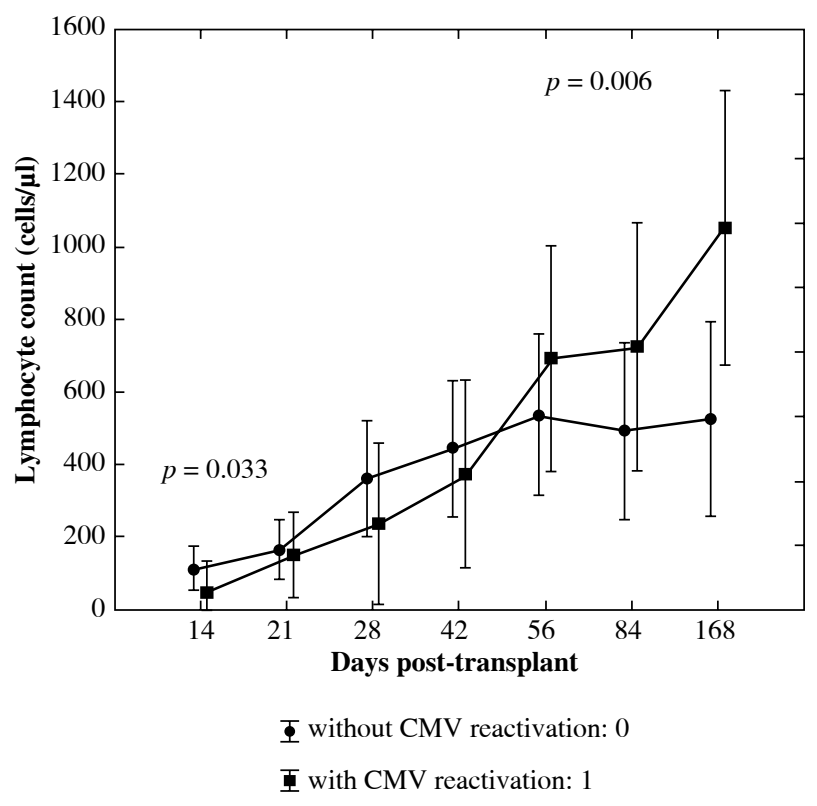

Fig. 3. Reconstitution of lymphocyte subsets after allogenic HSCT according to the occurrence of CMV reactivation - cytotoxic/suppressor $\mathrm{T}$ cells $\left(\mathrm{CD}^{+} \mathrm{CD}^{+}\right)$

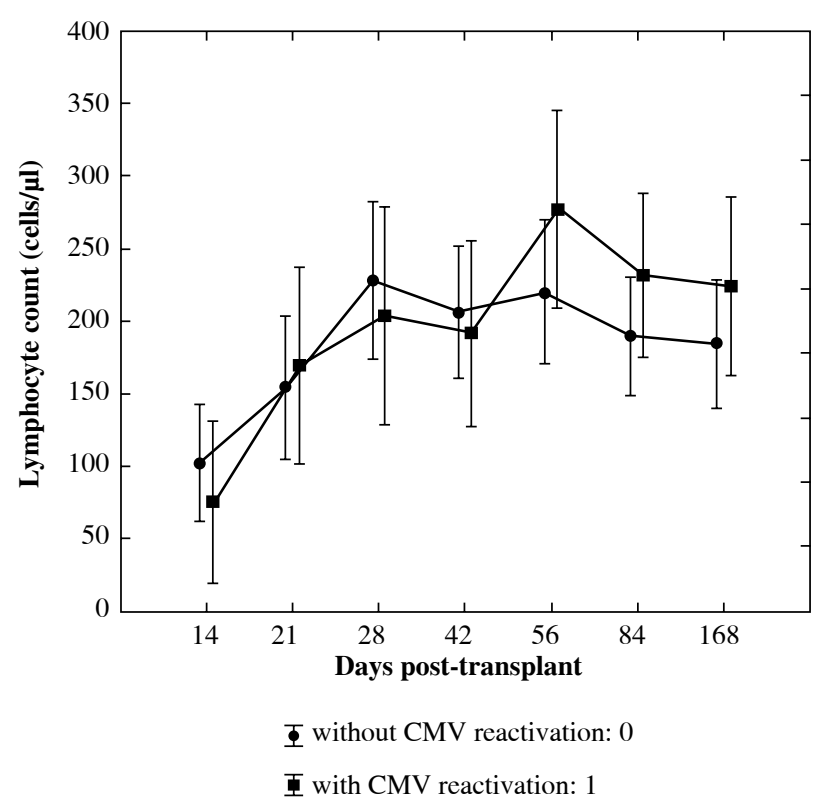

Fig. 5. Reconstitution of lymphocyte subsets after allogenic HSCT according to the occurrence of CMV reactivation - pan-NK lymphocytes $\left(\mathrm{CD}^{-}{ }^{-} \mathrm{CD}^{-} 6^{+}\right)$

phocyte values in the group with EBV reactivation comparing to the group without EBV reactivation were confirmed only in the case of pan-B lymphocytes $\left(\mathrm{CD} 19^{+}\right)$subpopulation on day $+21(p=0.005),+28(p=0.048)$ and lower on day $+84(p=0.002)$ post allo-HSCT (Fig. 6$)$.

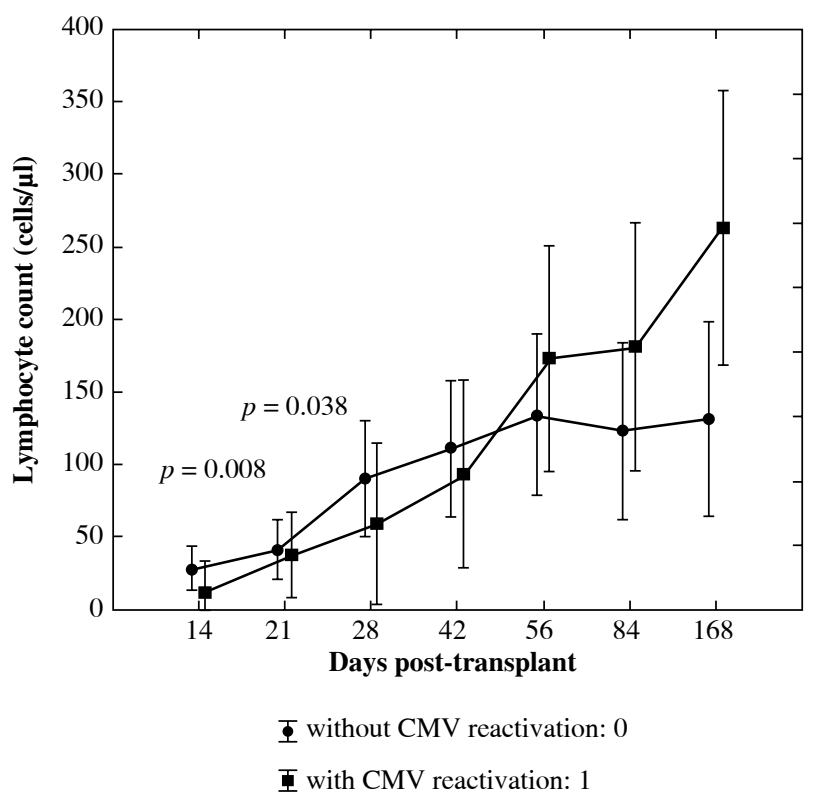

Fig. 4. Reconstitution of lymphocyte subsets after allogenic HSCT according to the occurrence of CMV reactivation - pan-B lymphocytes $\left(\mathrm{C} 19^{+}\right)$

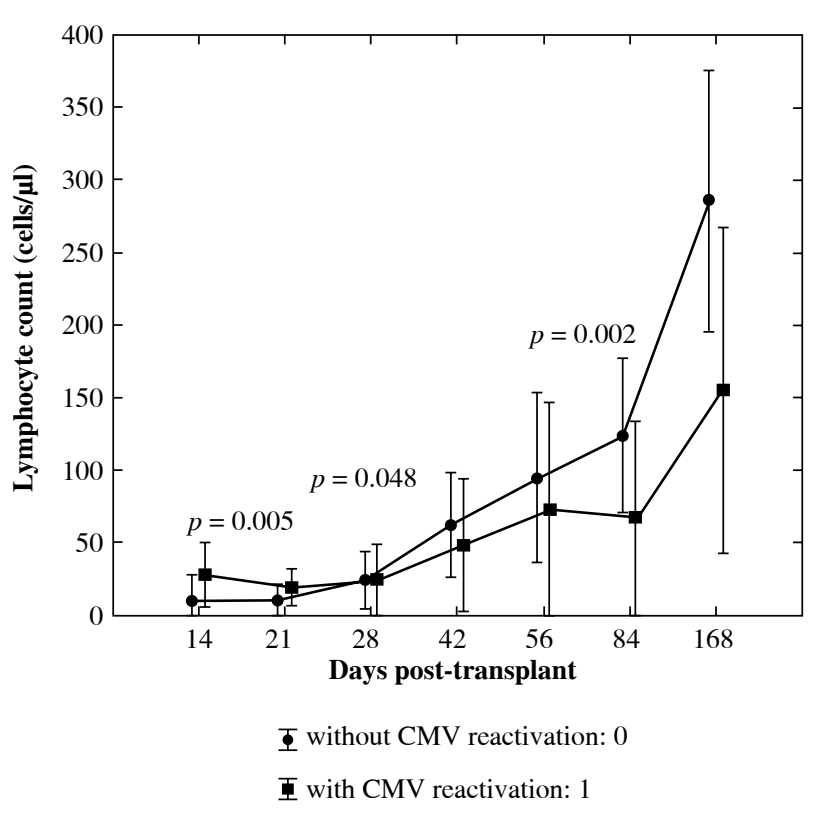

Fig. 6. Reconstitution of lymphocyte subsets after allogenic HSCT according to the occurrence of EBV reactivation - pan-B lymphocytes $\left(\mathrm{C} 19^{+}\right)$

To analyse the impact of: disease, donor, type of transplant, source of stem cells, conditioning regimen, CMV, and EBV serological status of donor and recipient, and recipients sex and age on the risk of CMV reactivation a qualitative $\mathrm{Chi}^{2}$ Pearson test was used. A significant dif- 
ference between the group without and with CMV reactivation was not confirmed in any of the above mentioned factors. However, an impact of CMV reactivation on the occurrence of the intestinal aGvHD was observed. This type of aGvHD was observed more frequently in the group with CMV reactivation compared with patients without CMV reactivation $(p=0.033)$. There was no significant impact of CMV reactivation on the overall incidence of other types of aGvHD as well as on the relationship between the degree of aGvHD (data not shown). However, patients with CMV and/or EBV reactivation (in total) have at least two times higher risk of aGvHD grade II-IV occurrence $\left(p=0.037\right.$ in $\chi^{2}$ Pearson test; $\mathrm{OR}=2.83 ; \mathrm{RR}=$ 2.05) (Table 3).

We also observed higher incidence of cGvHD in patients with CMV reactivation compared to the group without CMV reactivation $(p=0.016)$. In particular, cutaneous, intestinal, and hepatic cGvHD were observed more often in these patients $(p=0.012, p=0.018, p=0.035)$ without affecting the incidence of lung cGvHD, TTP or arthritis. CMV or EBV were detected respectively in 5/22 (23\%) and $7 / 31(23 \%)$ seronegative patients. In all cases the donors were seropositive for either CMV or EBV.

The impact of the donor (Fig. 7), and the type of transplant (Fig. 8) and GvHD prophylaxis (Fig. 9) on the frequency of EBV reactivation was also proven. EBV reactivation was observed more frequently in patients receiving transplants from matched unrelated donors (MUD, $p=$ $0.006)$, in particular MUDPBSCT ( $p=0.014)$, and while using ATG as GvHD prophylaxis ( $p=0.0001)$. The type of the disease, source of the stem cells, conditioning regimen, CMV and EBV serological status of the donor and recipient, occurrence and degree of GvHD, and sex or age of the recipient had no significant effect on the EBV reactivation. The $U$ Mann-Whitney test with continuity correction proved that the median age in children with CMV reactivation compared with the median age of patients without CMV reactivation was significantly higher $(p=0.016$, Fig. $10)$. No such relationship was observed when analysing the group of patients with and without EBV reactivation. No correlation between the amount of transplanted CD34 ${ }^{+}$ and $\mathrm{CD}^{+}$cells per $\mathrm{kg}$ of the recipient on $\mathrm{EBV}$ or $\mathrm{CMV}$ reactivation was observed.

\section{Discussion}

Here, we provide evidence that CMV reactivation leaves a long-lasting dynamic signature on the speed of immune recovery in paediatric HSCT recipients, which is in line with the Lugthart study [20]. Patients with poorer $\mathrm{CD}^{+}$cell reconstitution remain at risk of developing CMV reactivation. The infection itself stimulates $\mathrm{CD}^{+}$ cells to proliferate, which may lead to increased risk of aGvHD, and this was found in our study. The occurrence of aGVHD does not compromise, however, the prolonged marked relative and absolute expansion of $\mathrm{CD}^{+}$cells with effector memory and end stage effector phenotype, as proven by Lugthart et al. [20]. $\mathrm{CD}^{+}$cell expansion influences also pan- $\mathrm{T}$ cell $\left(\mathrm{CD}^{+}\right)$recovery in the late post-transplant period (i.e. six months post HSCT). Poorer early $\mathrm{CD}^{+}{ }^{+} \mathrm{T}$-cell reconstitution may increase the risk of CMV reactivation as well; however, these cells do not proliferate later in the course of recovery, probably as a result of CMV-mediated immune suppression and/or GvHD treatment. B cells remain low in patients with CMV reactivation partially due to coexisting EBV reactivation and its treatment with rituximab and partially due to GvHD therapy. NK cells recovery is not affected by viral reactivations. The typical pattern of CMV reactivation-mediated $\mathrm{CD}^{+}$cell recovery was not seen in patients with EBV reactivation (in our study and in the Lugthart study) and in patients with adenovirus reactivation in the Lugthart study [20]. Our observations remain in agreement with early studies by Würsch et al. in 1985, presenting the impact of CMV infection on the developing T-cell immunity in adult HSCT [21].

We could prove that the use of ATG in children with non-malignant disorders remains a significant risk factor for EBV reactivation post HSCT. Why is the risk higher in patients with non-malignant disorders in comparison with children with malignant diseases? ATG compromises the recovery of T cells, but not B cells, which are infected with EBV in seropositive patients. B cell recovery is generally impaired in patients with malignant disorders (due to pretreatment with conventional mostly intensive chemotherapy), which diminishes the risk of EBV reactivation. On the other hand, patients with non-malignant disorders experience usually prompt B cell recovery because they generally have not been pretreated with chemotherapy and/

Table 3. Number of patients with acute graft versus host disease (aGvHD) with or without CMV/EBV reactivation

\begin{tabular}{lccc}
\hline aGvHD status & CMV/EBV reactivation & $\begin{array}{c}\text { Without CMV/EBV } \\
\text { reactivation }\end{array}$ & Total aGvHD \\
\hline aGvHD grade 0-I $(\%)$ & $30(35)$ & $27(31)$ & $57(66)$ \\
\hline aGvHD grade II-IV $(\%)$ & $22(26)$ & $7(8)$ & $29(34)$ \\
\hline Total with or without reactivation $(\%)$ & $52(60)$ & $34(40)$ & $86(100)$ \\
\hline
\end{tabular}

$\chi^{2}$ Pearson test, $p=0.03725, O R=2.82, R R=2.06$ 


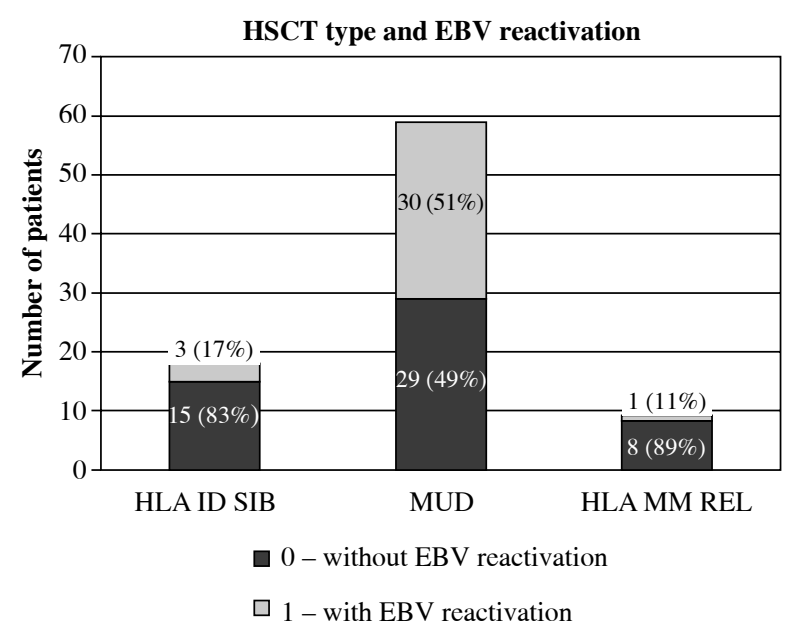

Fig. 7. Impact of HSCT type on EBV reactivation

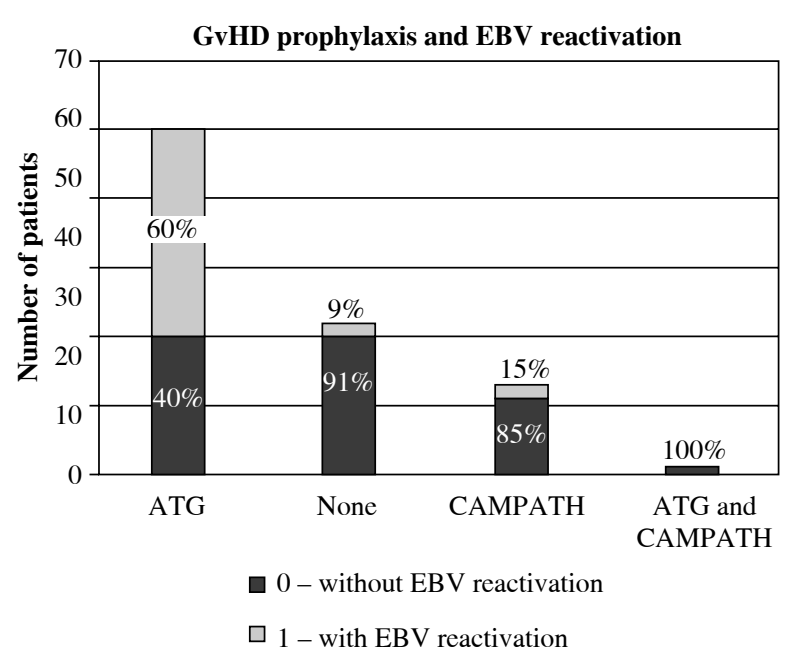

Fig. 9. Impact of GvHD prophylaxis on EBV reactivation

or immunosuppressive B cell depleting drugs. Whether we should exchange ATG with CAMPATH in the non-malignant setting requires further prospective studies. CAMPATH has proven to be effective in depleting both $\mathrm{T}$ and $\mathrm{B}$ cells, thus potentially reducing the risk of EBV reactivation [22]. In children with high risk of EBV reactivation, the preemptive administration of rituximab pre-transplant may be an effective prophylactic option. Rituximab depletes EBV-infected B cells and may delay B cell recovery, thus allowing T-cell recovery $[16,17]$. In our series PTLD developed in two out of 34 patients with reactivation. This potentially fatal complication should be prevented by all means, incl. a.m. rituximab both pre-transplant and post-transplant, when the first signs of EBV reactivation might be observed [23-25]. Whether EBV reactivation

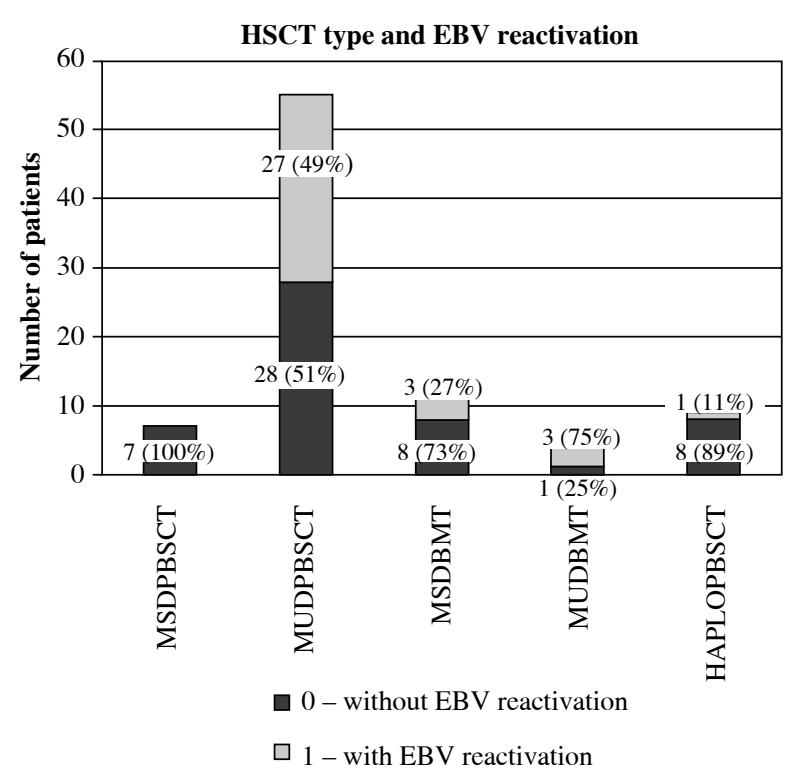

Fig. 8. Impact of HSCT type on EBV reactivation

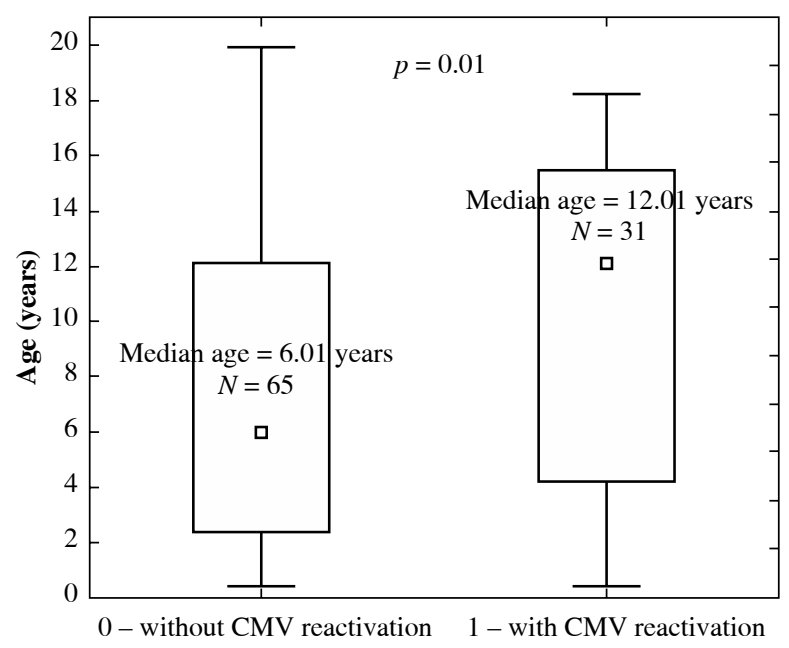

Fig. 10. Median age of children without CMV reactivation (0) compared with the median age of patients with CMV reactivation $(1), p=0.016$

(similar to CMV reactivation) influences the occurrence of GvHD, remains an open question. In our series of patients, we saw no impact of single EBV reactivation on GvHD. A recently published EBMT study by Styczynski et al. showed that positive EBV donor serostatus resulted in increased risk of both acute and chronic GvHD; however, we could not prove it in our study [26].

Our results highlight the importance of viral monitoring in the paediatric setting. To reduce the clinical impact of viral infections among these patients there is a need to 
identify tools for tailoring both the immunosuppressive and antiviral therapy.

\section{Conclusions}

In conclusion, impaired $\mathrm{T}$ cell recovery, in particular of the $\mathrm{CD}^{+} \mathrm{CD}^{+}$subset, favours $\mathrm{CMV}$ reactivation. $\mathrm{CMV}$ reactivation itself stimulates proliferation of $\mathrm{CD}^{+} \mathrm{CD}^{+}$ $\mathrm{T}$ cells, which may result in increased risk of acute intestinal Graft-versus-Host Disease. One should carefully search for both CMV seronegative or seropositive donor-recipient pairs and thoroughly monitor immune recovery. The use of ATG and prompt B-cell reconstitution favours EBV reactivation. In non-malignant disorders one may consider the use of CAMPATH-1H instead of ATG or preemptive administration of rituximab pre-transplant to minimise the risk of EBV reactivation.

The study was supported by grant No. 780 from Wroclaw Medical University.

The authors declare no conflict of interest.

\section{References}

1. Gratwohl A, Brand R, Frassoni F, et al. (2005): Cause of death after allogeneic haematopoietic stem cell transplantation (HSCT) in early leukaemias: an EBMT analysis of lethal infectious complications and changes over calendar time. Bone Marrow Transplant 36: 757-769.

2. Buhlmann LS, Buser AS, Cantoni N, et al. (2011): Lymphocyte subset recovery and outcome after T-cell replete allogeneic haematopoietic SCT. Bone Marrow Transplant 46: 1357-1362.

3. Kałwak K, Gorczyńska E, Toporski J, et al. (2002): Immune reconstitution after haematopoietic cell transplantation in children: immunophenotype analysis with regard to factors affecting the speed of recovery. Br J Haematol 118: 74-89.

4. Kałwak K, Porwolik J, Mielcarek M, et al. (2010): Higher CD34(+) and CD3(+) cell doses in the graft promote longterm survival, and have no impact on the incidence of severe acute or chronic graft-versus-host disease after in vivo $\mathrm{T}$ cell-depleted unrelated donor haematopoietic stem cell transplantation in children. Biol Blood Marrow Transplant 16: 388-401.

5. Kim DH, Kim JG, Sohn SK, et al. (2004): Clinical impact of early absolute lymphocyte count after allogeneic stem cell transplantation. Br J Haematol 125: 217-224.

6. Keever CA, Small TN, Flomenberg N, et al. (1989): Immune reconstitution following bone marrow transplantation: comparison of recipients of $\mathrm{t}$-cell depleted marrow with recipients of conventional marrow grafts. Blood 73: 1340-1350.

7. Wingard JR (1999): Opportunistic infections after blood and marrow transplantation. Transpl Infect Dis 1: 3-20.

8. Kim SY, Lee DG, Kim MS, et al. (2008): The influence of infection early after allogeneic stem cell transplantation on the risk of leukemic relapse and graft-versus-host disease. Am J Hematol 83: 784-788.

9. Boeckh M, Nichols WG, Papanicolaou G, et al. (2003): Cytomegalovirus in haematopoietic stem cell transplant recipients:
Current status, known challenges, and future strategies. Biol Blood Marrow Transplant 9: 543-558.

10. Ljungman P, Griffiths P, Paya C (2002): Definitions of cytomegalovirus infection and disease in transplant recipients. Clin Infect Dis 34: 1094-1097.

11. Styczynski J, Einsele H, Gil L, Ljungman P (2009): Outcome of treatment of Epstein-Barr virus-related post-transplant lymphoproliferative disorder in haematopoietic stem cell recipients: a comprehensive review of reported cases. Transpl Infect Dis 11: 383-392.

12. Ocheni S, Kroeger N, Zabelina T, et al. (2008): EBV reactivation and post transplant lymphoproliferative disorders following allogeneic SCT. Bone Marrow Transplant 42: 181-186.

13. Micallef IN, Chhanabhai M, Gascoyne RD, et al. (1998): Lymphoproliferative disorders following allogeneic bone marrow transplantation: the Vancouver experience. Bone Marrow Transplant 22: 981-987.

14. Faye A, Vilmer E (2005): Post-transplant lymphoproliferative disorder in children: incidence, prognosis, and treatment options. Paediatr Drugs 7: 55-65.

15. Styczynski J, Reusser P, Einsele H, et al. (2009): Management of HSV, VZV and EBV infections in patients with hematological malignancies and after SCT: guidelines from the Second European Conference on Infections in Leukemia. Bone Marrow Transplant 43: 757-770.

16. Faraci M, Caviglia I,Morreale G, et al. (2010): Viral-load and B-lymphocyte monitoring of EBV reactivation after allogeneic hemopoietic SCT in children. Bone Marrow Transplant 45: 1052-1055.

17. Masjosthusmann K, Ehlert K, Eing BR, et al. (2009): Delay in B-lymphocyte recovery and function following rituximab for EBV-associated lymphoproliferative disease early post-allogeneic haematopoietic SCT. Bone Marrow Transplant 43: 679-684.

18. Gärtner BC, Schäfer H, Marggraff K, et al. (2002): Evaluation of use of Epstein-Barr viral load in patients after allogeneic stem cell transplantation to diagnose and monitor posttransplant lymphoproliferative disease. J Clin Microbiol 40: 351-358.

19. Bacigalupo A, Ballen K, Rizzo D, et al. (2010): Defining the intensity of conditioning regimens: working definitions. Biol Blood Marrow Transpl 15: 1628-1633.

20. Lugthart G, van Ostaijen-Ten Dam MM, Jol-van der Zijde CM, et al. (2014): Early cytomegalovirus reactivation leaves a specific and dynamic imprint on the reconstituting $\mathrm{T}$ cell compartment long-term after haematopoietic stem cell transplantation. Biol Blood Marrow Transplant 20: 655-661.

21. Würsch AM, Gratama JW, Middeldorp JM, et al. (1985): The effect of cytomegalovirus infection on T lymphocytes after allogeneic bone marrow transplantation. Clin Exp Immunol 62: 278-287.

22. Anderson AE, Lorenzi AR, Pratt A, et al. (2012): Immunity 12 years after alemtuzumab in RA: CD5* B-cell depletion, thymus-dependent $\mathrm{T}$-cell reconstitution and normal vaccine responses. Rheumatology (Oxford) 51: 1397-1406.

23. Styczynski J, Reusser P, Einsele H, et al. (2008): Management of HSV, VZV and EBV infections in patients with hematological malignancies and after SCT: guidelines from the Second European Conference on Infections in Leukemia. Bone Marrow Transplant 43: 757-770.

24. Gil L, Styczyński J, Komarnicki M, et al. (2012): Strategy of pre-emptive management of Epstein-Barr virus post-trans- 
plant lymphoproliferative disorder after stem cell transplantation: results of European transplant centers survey. Contemp Oncol (Pozn) 16: 338-340.

25. Styczynski J, Gil L, Tridello G, et al. (2013): Response to rituximab-based therapy and risk factor analysis in Epstein Barr Virus-related lymphoproliferative disorder after haematopoietic stem cell transplant in children and adults: a study from the Infectious Diseases Working Party of the European Group for Blood and Marrow Transplantation. Clin Infect Dis 57: 794-802.

26. Styczynski J, Tridello G, Gil L, et al. (2016). Impact of donor epstein-barr virus serostatus on the incidence of graft-versushost disease in patients with acute leukemia after haematopoietic stem-cell transplantation: a study from the acute leukemia and infectious diseases working parties of the European Society for Blood and Marrow Transplantation. J Clin Oncol 34: 2212-2220. 